guished from a pure liquid by seeing whether the rings continue to move after the constant speed is reached.

(3) With concentrated solutions, the effects are more complicated, but they need not concern us here.

Now it is easy to show that an associated liquid is but a special case of miscible liquids, and as such is subject to all the osmotic laws. If we select a liquid which is but slightly associated it would come under category (2), and, on centrifuging, the two sets of molecules would, in general, be temporarily separated; and if we know from other sources their relative concentration, valuable light on the type of association might be obtained.

It may be pointed out that the sensitiveness of the mothod is, among other things, proportional (approximately) to the depth of liquid under examination; thus using a long column and a suitable optical system it might be possible to separate isotopes which are not otherwise separable.

As bearing on the whole subject I would direct attention to a paper by the late Dr. C. V. Burton and me on the "osmotic theory of solutions" (Phil. Mag., 1909, ment between the term values (see Table I.) of certain band groups of the many lined spectrum of $\mathbf{H}_{2}$ which he analysed (Proc. Roy. Soc., 113, 400; 1926) and the triplet system of the atomic helium spectrurn. In the case of $\mathrm{He}_{2}$ and $\mathrm{H}_{2}$ the analogy can be carried further in detail, due to the fact that under the usual conditions of excitation the band groups of the triplet system of $\mathrm{He}_{\mathrm{e}_{2}}$ ("Main-series') and the corresponding groups in the many lined spectrum of hydrogen (Fulcher bands, etc., see Proc. Roy. Soc., 113, 368 ; 1926) appear in greater intensity than the band groups of the singlet system of $\mathrm{He}_{2}$ ('Second series') and the corresponding groups of the many-lined spectrum of $\mathrm{H}_{2}$. Furthermore, there appear relatively many lines in the $Q$-branches of the band spectrum of $\mathrm{He}_{2}$ and in the many-lined spectrum of $\mathrm{H}_{2}$, while the $P$ - and $R$-branches fade out with comparatively low rotational quantum numbers.

Table I. gives a summary of the arrangement and magnitude of such of the electronic terms (in effective quantum numbers) of the spectra of $\mathrm{He}_{2}, \mathrm{He}$, and $\mathrm{H}_{2}$ as have been analysed.

The existence of the well-known $2^{3} \mathrm{~S}$ state in the

TABLIE I.

Observed Effective Quantum Numbers of Electronic States in Helium and Hydrogen.

\begin{tabular}{|c|c|c|c|c|c|c|c|c|c|c|c|c|c|c|c|}
\hline & \multicolumn{8}{|c|}{ Triplet System. } & \multicolumn{7}{|c|}{ Singlet System. } \\
\hline & \multicolumn{3}{|c|}{$\mathrm{He}_{2}$-Molecule. } & \multicolumn{3}{|c|}{ He-Atom. } & \multicolumn{2}{|c|}{$\mathbf{H}_{2}$-Molecule. } & \multicolumn{2}{|c|}{$\mathrm{He}_{2}-$ Molecule. } & \multicolumn{3}{|c|}{ He-Atom. } & \multicolumn{2}{|c|}{$\mathbf{H}_{2}$-Molecule. } \\
\hline & $S$. & $P$. & $D$. & $S$ & $P$. & $D$. & $S$. & $P$. & $S$ & $P$. & $S$ & $P$. & $D$. & $S$ & $P$. \\
\hline $\begin{array}{l}1 \\
2 \\
3 \\
4 \\
5 \\
6\end{array}$ & $\begin{array}{c}1 \cdot 788 \\
2 \cdot 810 \\
3 \cdot 818 \\
\ldots\end{array}$ & $\begin{array}{c}(1.928)^{*} \\
2.928 \\
3.928 \\
4.928 \\
5.927 \\
\text { etc. }\end{array}$ & $\begin{array}{c}3 \cdot \ddot{013} \\
\ldots \\
\cdots\end{array}$ & $\begin{array}{c}1 \cdot 689 \\
2 \cdot 697 \\
3 \cdot 700 \\
4 \cdot 701 \\
\ldots\end{array}$ & $\begin{array}{c}1.937 \\
2 \cdot 933 \\
3 \cdot 932 \\
4 \cdot 932 \\
\text { etc. }\end{array}$ & $\begin{array}{c}2 \cdot 997 \\
3 \cdot 997 \\
4 \cdot 997 \\
.\end{array}$ & $\begin{array}{c}1 \cdot 934 \\
\ldots \\
\ldots \\
\ldots\end{array}$ & $\begin{array}{c}(1.928)^{*} \\
2.937 \\
3.939 \\
4.941 \\
5.941 \\
\text { ete. }\end{array}$ & $\begin{array}{c}0.744 \\
1.853 \\
\quad \ldots \\
\ldots \\
\ldots\end{array}$ & $\begin{array}{c}\ddot{0} \\
2 \cdot 964 \\
3 \cdot 965 \\
4 \cdot 966 \\
5 \cdot 964\end{array}$ & $\begin{array}{c}0 \cdot 744 \\
1 \cdot 850 \\
2 \cdot 857 \\
3.858 \\
\ldots \\
\ldots\end{array}$ & $\begin{array}{l}2009 \\
3.011 \\
4 \cdot 011 \\
\text { etc. }\end{array}$ & $\begin{array}{c}\ddot{ } \\
2 \cdot 998 \\
3 \cdot 998 \\
\ldots \\
\cdots\end{array}$ & $\begin{array}{c}0.919 \\
1.919 \\
(2.920)^{*} \\
\ldots \\
\ldots\end{array}$ & $\begin{array}{c}1.695 \\
(2.695)^{*} \\
\cdots \\
\cdots\end{array}$ \\
\hline
\end{tabular}

* Calculated.

p. 598 ; there are printer's errors on p. 612), which may help in the matter ; indeed, the optical method indicated above was devised by us so as to continue the centrifuging experiments mentioned therein; but I am not in a position to pursue the research any further.

Since writing the above I have seen Messrs. Raman and Krishnan's letter on "The Maxwell Effect in liquids" (Nat'dre, Nov. 19). It would be interesting to see whether the 'optical' centrifuge could be used to test their theory; if, however, it takes time for asymmetric molecules to orient themselves, it will be difficult to distinguish between this effect and that caused by association.

Berkeley Castle,

BERKELEY.

Gloucestershire.

\section{Absorption Experiments on Excited Molecular Hydrogen.}

RECENT investigations on the band spectrum of helium and the many-lined spectrum of hydrogen seem to show that there is a far-reaching analogy between the arrangement and location of the electronic states of the term systems of the spectra of $\mathrm{He}$, $\mathrm{He}_{2}$, and $\mathrm{H}_{2}$. For the spectra of $\mathrm{He}$ and $\mathrm{He}_{2}$ this analogy has been discussed by Mullikan (Proc. Nat. Acad. Sci., 12, 158; 1925) and extended by the author (Proc. Nat.Acad.Sci., 13, $213 ; 1927$ ). In regard to the correspondence between the electronic states of $\mathrm{H}_{\theta}$ and $\mathrm{H}_{2}$, Richardson demonstrated a remarkable agree-

No. 3032, VoL. 120] helium spectrum, and the fact that intercombinations between the triplet and singlet systems mentioned in Table J. have not been observed (except for the combination $\lambda 591.56$ found by Lyman in the helium spectrum, which he classifies as the transition $\left.1^{1} S_{0}-2^{3} P_{1}\right)$ led me to look for a corresponding metas. table state of $\mathrm{H}_{2}$ by absorption experiments in excited molecular hydrogen. The experimental conditions in the emission tube were chosen so as to produce the many-lined spectrum in particular. In the absorption tube a weak electric excitation of the hydrogen gas was used in order to excite any existing metastable states, that these might be the initial states of absorption for the radiation of the $\mathbf{H}_{2}$-molecules in tho omission tube. If there is any absorption it should be shown by self-reversal of certain band lines.

The absorption measurements which have been made over the whole region of the visible manylined spectrum seem, indeed, to have the expected result. A number of intense lines, which are not distributed over the whole spectrum but are located in definitely bounded regions, distinctly show selfreversal, while other intense lines scattered over the whole spectrum do not show any absorption. The study of the series relations of the reversed lines is in progress. After further experiments the results will be published elsewhere in detail. It is intended to extend the absorption experiments to excited molecular helium.

Jefferson Physical Laboratory, Cambridge (Mass.), Sept. 30. 